\title{
Awareness of prostate cancer among patients and the general public: results of an international survey
}

\author{
JM Fitzpatrick ${ }^{1}$, RS Kirby ${ }^{2}$, CL Brough ${ }^{3}$ and AL Saggerson ${ }^{4}$ \\ ${ }^{1}$ Department of Surgery, Mater Misericordiae Hospital and University College Dublin, Dublin, Ireland; ${ }^{2}$ The Prostate Centre, \\ London, UK; ${ }^{3}$ GlaxoSmithKline, London, UK and ${ }^{4}$ Galliard Healthcare Communications, London, UK
}

\begin{abstract}
The objective of this study was to assess the level of awareness of prostate cancer (PCa) among the general public and PCa patients in Europe and North America. A survey was undertaken across four European countries (UK, Germany, Italy and Spain), and across the United States and Canada in late 2007. In total, 1008 men with PCa and their partners (the 'prostate sample'), and 911 men without PCa and their partners (the 'well sample') participated in the survey, all aged $\geqslant 50$ years. Interviews were conducted through telephone, pen and paper, and online. Many people surveyed (53\%) thought that breast cancer is more common than PCa. Moreover, 1 in 10 people from the well sample (10\%) thought that PCa affects both men and women. When the prostate sample was asked about their perceived level of risk of PCa before diagnosis, $50 \%$ believed that they/their husband or partner were previously at low or very low risk, before they were diagnosed. Awareness of the major risk factors for PCa (age and family history) was generally good, but respondents were less clear about the role of other potential factors, such as smoking and drinking alcohol. This international survey, thought to be largest of its type, shows that although patient and public awareness of PCa is generally satisfactory, there is still a considerable lack of clarity about PCa risk factors, and a danger for people to underestimate their own/their partner's perceived risk for PCa. Programmes to responsibly educate and inform men and their partners about risk factors, prevalence and screening tools for PCa are required.

Prostate Cancer and Prostatic Diseases (2009) 12, 347-354; doi:10.1038/pcan.2009.30; published online 21 July 2009
\end{abstract}

Keywords: awareness; risk; patients; epidemiology; survey

\section{Introduction}

Prostate cancer (PCa), the most frequently diagnosed cancer in men, ${ }^{1,2}$ is diagnosed in almost 2000 men each day worldwide, and one man is estimated to die from the disease every 2 min. $^{3}$ With 1 in 6 men in the United States $^{4}$ and 1 in 11 men in Europe ${ }^{5}$ estimated to be diagnosed with PCa at some point in their lifetime, the disease has been said to be already approaching epidemic proportions. ${ }^{2}$ Furthermore, because threequarters of all men diagnosed with $\mathrm{PCa}$ are aged $\geqslant 65$ years ${ }^{3}$ and with an aging population in many regions of the world, the prevalence of the disease is likely to increase, with concomitant socioeconomic and medical implications. ${ }^{5}$

Given this worrying situation, it is even more concerning that men who seek knowledge about PCa are faced with a lack of clarity and consensus in some areas, specifically with regard to individual risk,

Correspondence: Professor J Fitzpatrick, Department of Surgery, Mater Misericordiae Hospital and University College Dublin, 47 Eccles Street, Dublin 7, Ireland.

E-mail: jfitzpatrick@mater.ie

Received 14 April 2009; revised 16 June 2009; accepted 16 June 2009; published online 21 July 2009 perceived risk factors, screening and treatment options. Some of this uncertainty might likely stem from a significant neglect of PCa compared with other cancers, in particular breast cancer, both in the media and in terms of clinical research, even though both the diseases have a similar prevalence. ${ }^{6,7}$ A recent survey of the media revealed that there are $40 \%$ more articles focused on breast cancer compared with $\mathrm{PCa}{ }^{8}$ Moreover, the considerable 'research gap' between $\mathrm{PCa}$ and breast cancer is reflected by the fact that there are currently more than 1000 active trials for the treatment of breast cancer compared with only around half that number for PCa. ${ }^{9}$

Owing to the long gestation period of $\mathrm{PCa}$, there is significant interest in emerging therapies that reduce the risk of developing the disease. A greater understanding of the potential role of PCa risk reduction has been provided by a number of trials, namely the Prostate Cancer Prevention Trial (PCPT) ${ }^{10,11}$ which showed that intervention with finasteride therapy in men decreases the period prevalence of PCa. In addition, the REduction by DUtasteride of prostate Cancer Events (REDUCE) ${ }^{12,13}$ trial showed that dutasteride reduces the risk of PCa in men at increased risk of the disease. Further investigations may continue to shed light on the role of pharmaceutical interventions in PCa risk reduction. 
In view of the emerging and new interventions for $\mathrm{PCa}$, in this report, we ask what current level of awareness exists among men and their partners about PCa: the disease itself (prevalence, mortality and disease characteristics), perceived risk factors and screening. Which aspects of PCa awareness give cause for concern? Moreover, do levels of awareness regarding these factors change on diagnosis of PCa?

To our knowledge, although there have been notable and important national surveys on PCa awareness, ${ }^{14-16}$ there has been no large-scale international research on public awareness of risk factors for PCa among those affected by the disease (patients and their partners) and among the general public since the survey results published by Schulman et al. ${ }^{17}$. This report surveyed six European countries (France, Germany, Italy, Spain, Sweden and the United Kingdom) and the United States, and included 1400 men and women, aged between 40 and 70 years. Therefore, in what we believe is the most recent and a unique investigation of its kind and scale, we sought to understand current perceptions and awareness of $\mathrm{PCa}$ among the PCa patients and the general public in six countries: Canada, Germany, Italy, Spain, the United Kingdom and the United States.

Here, we report the results of this international survey, designed to evaluate awareness of $\mathrm{PCa}$, that is, its diagnosis, prevalence and risk factors. Men both with and without PCa, and their partners, above the age of 50 years, were questioned. The structure of the current survey is similar to that of Schulman et al. ${ }^{17}$ in the countries sampled and the types of questions asked (that is, concerning risk factors and prevalence for $\mathrm{PCa}$ ). Therefore, although direct comparisons cannot be drawn between studies, the present survey findings allow us to learn about not only current levels of PCa awareness among the patients and the public, but also about the progress made in public awareness of PCa since 2003.

\section{Materials and methods}

\section{Participants}

GlaxoSmithKline commissioned the independent research company Ipsos MORI (London, UK) to conduct the survey in 2007. Ipsos is a leading multi-research company with global reach, whose operatives have extensive experience in collecting data from international sources. The core research objectives were to measure the awareness of the severity and implications of PCa, assess knowledge of PCa compared with other cancers, and to understand how the public perceive and contextualize their level of risk.

\section{Methodology}

Interviews were conducted through telephone, pen and paper and online, in UK, Germany, Italy, Spain, the United States and Canada between October and December 2007. The survey sample consisted of 1008 men with PCa and their partners (the 'prostate cancer sample') and 911 men without PCa and their partners (the 'well sample'). All participants were aged $\geqslant 50$ years. Although there were slightly more participants in the PCa sample than in the well sample, the number of
PCa responders from each country were about equal. Information about the mean age, education or comorbidities of the patients was not obtained.

In terms of information collection, all the well samples were surveyed through telephone, whereas the PCa samples in Germany, Italy, Spain were surveyed through pen and paper, the PCa sample in the United Kingdom through telephone and the PCa samples in the United States and Canada were questioned online.

\section{Questionnaire}

The questionnaire used for the survey is given in the Appendix A. The questions were both prompted and open ended.

The questions used in the survey were rigorously drafted on the basis of advice from (including but not limited to):

- A select group of physicians, interviewed at the European Association of Urology meeting in 2007.

- A selection of PCa patient group's representatives.

Questions were then screened for clarity and balance by Ipsos MORI.

The questions were then translated for use in each of the individual countries. The questions were tested by pilots in each market, which threw up any areas of confusion, which were then refined further.

Where appropriate, questions were asked to all respondents (question sample is detailed with each question in appendix and in Results below).

\section{Results}

Results have been split out, where differences pertain, for the well sample and the PCa sample, and according to country surveyed.

\section{$P C a$ prevalence}

The total sample was asked, 'Which one of the following is the most common form of cancer in men in this country?' from a list of common cancer types including bladder, lung and colorectal cancer (Q1). All groups said that they thought $\mathrm{PCa}$ was the most common cancer type over and above the other options, with $65 \%$ of the total sample giving this response.

When asked, 'Which one of the following is more common in this country, breast cancer in women or PCa in men?' (Q2), 53\% of the total sample said breast cancer and $18 \%$ said PCa (Figure 1). Of note, $63 \%$ of the well sample said that they thought breast cancer was more common than PCa compared with $43 \%$ of the PCa sample.

\section{PCa mortality}

The well sample were asked 'In your opinion, among those who have ever suffered from $\mathrm{PCa}$, what percentage of them actually die from the disease, as opposed to old age or another cause of death?' (Q3). The well sample questioned believed that around one-third of men (32\%) will die from PCa. In Italy, they were more optimistic than this, giving an average figure of $26 \%$. 


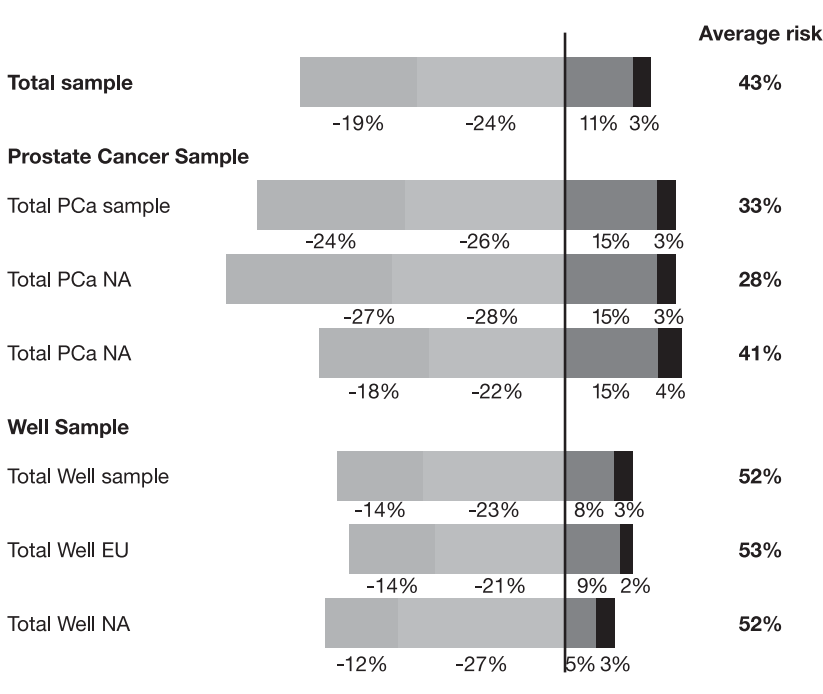

Figure 1 Perceptions of respondents (grouped by sample) when asked if they thought prostate cancer or breast cancer was more common in their country. Left-hand bars: breast cancer is more common; right-hand bars: prostate cancer is more common. The color reproduction of this figure is available in the html full-text version of the paper.
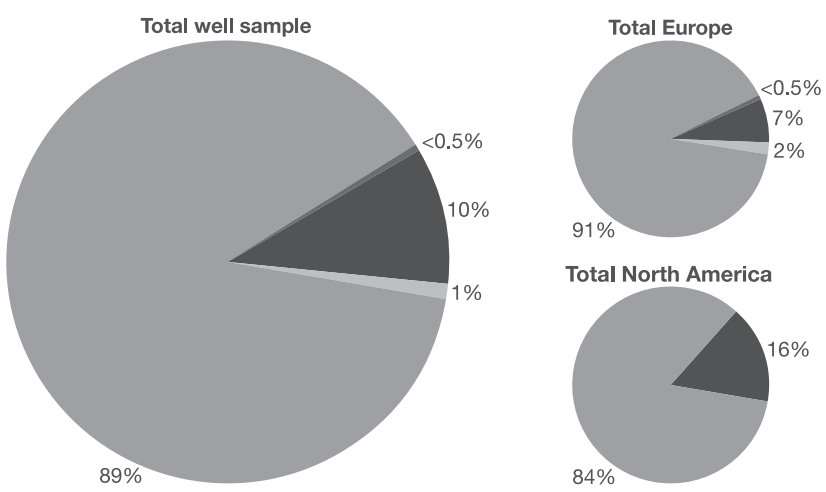

Total North America

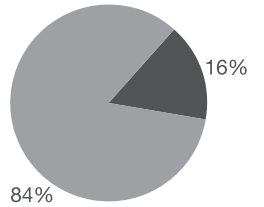

Figure 2 Responses of participants (well sample) when they were asked 'who does prostate cancer affect: men, women or both?' Very light shading: don't know; light shading: only men; medium shading: only women; dark shading: both. The color reproduction of this figure is available in the html full-text version of the paper.

\section{PCa disease characteristics}

The well sample was asked, 'As far as you know, does PCa only affect "men", only affect "women" or both?" (Q4). Overall, 1 in 10 people (10\%) said that they thought PCa affects both men and women (Figure 2). In North America, this figure was higher, at $16 \%$. In Europe, $7 \%$ of people thought that PCa affects men and women, 2\% said 'Don't know' and $0.5 \%$ said that they thought that PCa only affects women.

\section{PCa signs and risk factors}

To attempt to understand the participant's contextualization of PCa risk, the prostate sample was asked 'Before you were diagnosed with PCa, how much at risk did "you" / "your husband/partner" believe "you were"/ "he was" of developing PCa? To assess this among the well sample, they were asked 'Generally speaking, how much at risk of PCa do you believe "you are" /"your
Figure 3 Perceptions of prostate cancer risk among respondents (split by sample). Far left bar: very low risk; middle left: low risk; middle right: quite high risk; far right: very high risk. The color reproduction of this figure is available in the html full-text version of the paper.

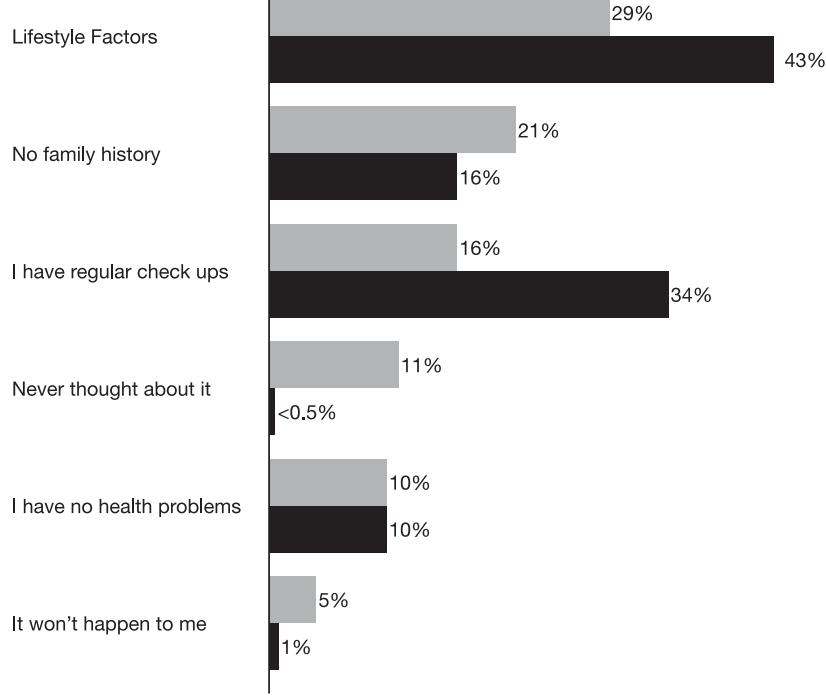

Figure 4 Reasons cited for perceived low prostate cancer risk among those who answered 'low' to the previous question (split by sample). Light bars: prostate cancer sample; dark bars: well sample. The color reproduction of this figure is available in the html full-text version of the paper.

husband/partner is" compared with an average man of the same age?' All groups were given the options 'Very low, low, quite high risk, very high risk' (Q5). Among the total sample, almost half (43\%) believed that this risk was low or very low (Figure 3 ). Among the prostate sample, $50 \%$ believed that they/their husband or partner, were at low or very low risk before they were diagnosed.

As a follow-up to this question, those in the total sample who responded as 'low' were asked 'And why do you think your risk is low?' (Q6), 26\% of the total sample answered without any firm response (Figure 4)-11\% said 'Never thought about it,' $10 \%$ said 'I have no health problems' and 5\% said 'It won't happen to me.' 
The main, prompted responses to this question by both groups were 'lifestyle factors', 'no family history' and 'I have regular checkups'. Of note, $21 \%$ of the prostate sample cited family history as a reason for why they thought their/their partner's risk was high/low compared with $16 \%$ of the well sample.

Of those who had answered 'high' to question Q5, the main reason cited by the total sample for their believing they had this level of risk was 'genetic reasons', for example, 'father had it $(42 \%)$ '. The main reason cited by the well sample was 'age', that is, 'being older $(25 \%)^{\prime}$.

The total sample was asked 'In your opinion, which of the following factors, if any, could mean that a person becomes more likely to develop PCa?' (Q7). Age (84\%) and family history (77\%) were the most popular responses, whereas 43 and $36 \%$ of people thought that smoking and drinking, respectively, were factors that could increase a person's risk of PCa.

\section{PCa screening}

Question 8 asked the total sample: 'And in your opinion, which of the following, if any, can men do to reduce their likelihood of developing PCa?' The list of prompted options to this question included 'exercise regularly, eat plenty of fruit and vegetables, have regular checkups / screening, not carrying a mobile phone in their pockets'. Most people (94\%) thought that the main thing that men can do to reduce their prostate PCa risk is to go for regular screening. This was closely followed by healthy diet $(77 \%)$ and regular exercise $(66 \%)$. Of note, $14 \%$ of people who were asked this question thought that not carrying a mobile phone in their pocket would reduce men's risk of PCa.

In Germany specifically, nearly one-third of those questioned believed that carrying a mobile phone in their pocket could increase a person's risk of developing PCa.

When asked from a list of options (Q9; including stool sample test, sperm count, PSA test, CRP (C-reactive protein) test), 'Which, if any, of the following is used to screen for PCa?', even when prompted, $31 \%$ did not cite the PSA test.

\section{Discussion}

The current survey results indicate that awareness of PCa among European and North American men and their partners is generally good, but there are some specific areas of concern. Moreover, the survey reveals many interesting disparities between the well sample and the PCa sample, and also some specific country differences.

It is noteworthy that people's awareness of PCa, its prevalence and risk factors is generally good, and has improved somewhat since the time of the last published survey of this kind of which we are aware-that reported by Schulman et al. ${ }^{17}$

\section{Awareness of PCa prevalence}

With respect to awareness of the prevalence of $\mathrm{PCa}$, the majority of people $(65 \%)$ knew that PCa is the most common cancer in men. In this respect, people's knowledge is accurate, as PCa is the most commonly diagnosed cancer in men in Europe, and in the United States and Canada. ${ }^{1,3,4}$

These findings point to considerable improvement in PCa awareness since the report by Schulman et al. ${ }^{16}$ in 2003 , in which it was revealed that only $39 \%$ of men and $28 \%$ of women spontaneously mentioned PCa when asked what types of cancer they were aware of compared with $79 \%$ of people who mentioned breast cancer. The present findings indicate that many people questioned knew, albeit when prompted, that PCa is the most common form of cancer in men.

However, it is interesting that the majority of people (53\%) think that breast cancer is more common than PCa, especially as PCa is actually more common than breast cancer in one-third of the countries surveyed, that is, in the United States and Italy., ${ }^{1,6}$ the remaining countries surveyed, the prevalence of PCa is roughly equivalent to that of breast cancer, with age-standardized PCa incidence rates for Germany, the United Kingdom, Spain and Canada ranging from an average of 93 to 124 cases per 100000 men. ${ }^{1,6}$ These findings are concordant with those of a recent survey in the United States conducted by the Prostate Cancer Foundation (2006), ${ }^{14}$ which revealed that nearly three-quarters of 1572 people questioned believed that PCa was less common among men than breast cancer is among women.

It is interesting that the levels of awareness about the incidence of $\mathrm{PCa}$ in the present study is lowest in Spain and in Germany compared with that in other European countries questioned, where 62 and 57\%, respectively, think that breast cancer is more common than PCa. In Spain, even among men with PCa, 66\% believed that breast cancer is more common than PCa.

The finding that breast cancer is thought to be more common than PCa is perhaps attributable to the more widespread publicity that breast cancer achieves compared with PCa. This fact may also be because of the etiology of the disease, as although breast cancer often causes deaths of women under the age of 45 years, ${ }^{7}$ PCa usually affects a rather different demographic, and most commonly affects men over the age of 50 years. ${ }^{2}$ However, whatever may be the cause for this disparity, it is undoubted that public education programmes should address this issue.

\section{Awareness of PCa mortality rates}

People's awareness of mortality rates was good, with those questioned believing that about one-third of men $(32 \%)$ with PCa will die from the disease, and this figure is fairly accurate. More than 200000 men each year die from PCa worldwide. ${ }^{1,2}$ Since 1995, the number of men dying from PCa in Europe has increased by $16 \% .^{1}$ This may be because of a large increase in the numbers of men reaching older ages-when men are more likely to develop PCa. ${ }^{5}$

\section{Awareness of PCa disease characteristics}

One of the most worrying findings from this survey was that 1 in 10 people $(10 \%)$ questioned think that PCa affects both men and women, and $1 \%$ of those questioned did not know whether it affects men or women. The United States and Canada had the highest proportion of people who believed that PCa affected men 
and women, at 15 and 17\%, respectively. It is, however, feasible that by the structuring of the question, that is, 'As far as you know, does PCa only affect "men", only affect "women" or both?' respondents understood that indeed, PCa does affect the quality of life of both men and women. Nevertheless, further investigation is warranted to clarify this issue.

\section{PCa signs and risk factors: age and family history}

The survey highlighted that people had a good knowledge of the most common risk factors for PCa, such as age $(84 \%)$ and family history $(77 \%)$. Therefore, when considering these factors alone, people's awareness of risk factors was accurate. A man's risk of developing PCa increases in proportion to his age, with about threequarters of all cases diagnosed being in men aged $\geqslant 65$ years. ${ }^{3}$ Moreover, family history is known to be a strong risk factor for $\mathrm{PCa} .{ }^{18}$ Having a first-degree relative with $\mathrm{PCa}$ significantly increases the risk of developing the disease compared with those having no first-degree relatives with the disease. ${ }^{18}$ For example, if you have a father or brother with Pca, you have two to three times the risk of developing the disease compared with those having no first-degree relative affected. If you have a father or brother with early onset PCa (which develops under the age of 60 years), you have a fourfold increase in risk compared with those having no first-degree relative affected. Finally, if you have more than one firstdegree relative with early onset $\mathrm{PCa}$, you have a ninefold increase in risk of developing $\mathrm{PCa} .{ }^{18}$

\section{PCa signs and risk factors: smoking and drinking}

However, although people's knowledge of age and family history was relatively good, the responses relating to smoking and drinking would seem to reflect the lack of clarity on the role of these factors. Of those questioned, 43 and $36 \%$ of people think that smoking and drinking, respectively, are risk factors for PCa. There is no clear role for these risk factors in the development of $\mathrm{PCa}^{2}$ However, as these factors have a large influence on the etiology of so many other diseases and cancer types, it is perhaps understandable that people have misconceptions about their influence on the development of PCa. People's understanding of the main risk factors for PCa seems to have improved considerably since the survey reported by Schulman et al. ${ }^{17}$ However, again, this is clearly an area in which public education and awareness programmes are required.

\section{PCa signs and risk factors: other issues mentioned}

It must be noted that while this survey did not include a prompted option to state race as a risk factor for $\mathrm{PCa}$, respondents were given the option to spontaneously cite other factors that they believed to be PCa risk factors. Despite this, it is interesting that none mentioned race as a risk factor, although recent estimates show that Black men have more than one-and-a-half times the risk for developing PCa than White men. ${ }^{19}$ Black men would therefore be a particularly salient target for disease education and awareness programmes.

Remarkably, $14 \%$ of people thought that they could reduce their risk of $\mathrm{PCa}$ by 'not carrying a mobile phone in their pocket'. In Germany, this figure was highest of all the countries surveyed, with nearly one-third (28\%) of those questioned believing that this was the case. No clear relationship has been established between the use of mobile phones and the development of cancer. ${ }^{20}$

PCa signs and risk factors: underestimating your own risk One of the most important and interesting finding from this survey was that many men may be underestimating their own risk of PCa and not contextualizing this risk. The survey revealed that many people $(43 \%)$ believe that their risk of $\mathrm{PCa}$ is low or very low-even many men with PCa believed they were at low risk before they were diagnosed. However, most men believe that they have an average (also 43\%) risk of developing PCa. Although difficult to quantify, the current consensus is that 1 in 6 men in the United States ${ }^{4}$ and 1 in 11 men in Europe ${ }^{5}$ will develop clinical PCa in their lifetime.

When the total sample was asked what their reasons for thinking they previously had a low level of the risk of developing PCa were, $26 \%$ said that they had either 'Never thought about it' or claimed 'I have no health problems' or believed 'It won't happen to me.'

\section{Screening for $\mathrm{PCa}$}

Most people think that the main thing that men can do to reduce their PCa risk is to go for regular screening (94\%). These data indicate that the survey participants had a very good awareness of screening. However, a note of caution should be added, as this finding may also point to some lack of clarity among the public concerning screening, and to a need for increased education. Although it is true that undergoing regular screening may allow identification of the precursors to PCa or diagnose PCa at early stages, thereby indirectly reducing a man's risk of developing the disease and/or reduced PCa-related mortality, clearly, screening does not reduce the risk of developing PCa per se. Moreover, the recently published ERSPC trial $^{21}$ results showed that screening decreased PCa-related mortality by $20 \%$; however, an increase in PCa over diagnosis and over treatment was observed. Further analyses are thus needed to determine the optimal screening interval in consideration of the PSA value at the first screening and of previously negative results on biopsy. ${ }^{21}$

It should be acknowledged that there were certain limitations to the survey, notably that no information about the education or the comorbidities of the patients was recorded. Moreover, although all participants were aged $\geqslant 50$ years, no break down of ages was available. This prevents stratification of the data by these factors, thereby limiting interpretations of the data in this way. Future surveys of this type should include recording of these data and may reveal findings of interest.

\section{Conclusions}

Since the report by Schulman et al. ${ }^{17}$, awareness of PCa seems to have improved somewhat, with respect to the disease itself (gestation time, prevalence and mortality rates), individual risk, perceived risk factors and screening. However, there are still a proportion of men and 
their partners, who seem to be confused about these PCa facts. Even more worrying is the finding of the survey that many men perceive their risk of developing PCa to be either low or average. Again, this is a clear area of concern, and as such a focus for future disease education and awareness programmes.

There also seems to be considerable disparity between the countries surveyed in terms of PCa awareness, particularly with regard to $\mathrm{PCa}$ prevalence and the disease itself (that is, who does PCa affect?). We would recommend that programmes to responsibly educate and inform men and their partners about PCa are urgently required to bridge the gap in awareness between $\mathrm{PCa}$ and other cancers, and that these awareness programmes are sufficiently sensitive to take into account these country-specific differences.

\section{Conflict of interest}

The survey reported in this paper was commissioned and funded by GlaxoSmithKline. Professor John Fitzpatrick is a consultant to GlaxoSmithKline, Claire L Brough is an employee of GlaxoSmithKline. Medical writing support in the preparation of this manuscript was provided by Anna L Saggerson and funded by GlaxoSmithKline. Professor Roger Kirby has no conflicts of interest.

\section{References}

1 Ferlay J, Autier P, Boniol M, Heanue M, Colombet M, Boyle P. Estimates of the cancer incidence and mortality in Europe in 2006. Ann Oncol 2007; 18: 581-592.

2 Bostwick DG, Burke HB, Djakiew D, Euling S, Ho SM, Landolph J et al. Human prostate cancer risk factors. Cancer 2004; 101 (Suppl): 2371-2490.

3 Parkin DM, Bray F, Ferlay J, Pisani P. Global cancer statistics 2002. CA Cancer J Clin 2005; 55: 74-108.

4 Jemal A, Siegel R, Ward E, Murray T, Xu J, Thun MJ. Cancer statistics, 2007. CA Cancer J Clin 2007; 57: 43-66.
5 Schulman C. The aging male: a challenge for urologists. Curr Opin Urol 2000; 10: 337-345.

6 Globocan 2002 database. http:/ / www-dep.iarc.fr/GLOBOCAN/ Table2 sel.htm.

7 Kamangar F, Dores GM, Anderson WF. Patterns of cancer incidence, mortality, and prevalence across five continents: defining priorities to reduce cancer disparities in different geographic regions of the world. J Clin Oncol 2006; 24: 2137-2150.

8 Number of stories containing the search terms 'prostate cancer' or 'breast cancer', years 2006-2007. Source: Google advanced news archive search.

9 Clinical Trials.gov. http://clinicaltrials.gov

10 Thomson IM, Ankerst DP, Chi C, Goodman PJ, Tangen CM, Lucia MS et al. Assessing prostate cancer risk: results from the Prostate Cancer Prevention Trial. J Natl Cancer Inst 2006; 98: 529-534.

11 Thompson IM. The influence of finasteride on the development of prostate cancer. N Engl J Med 2003; 349: 215-224.

12 Gomella L. Chemoprevention using dutasteride: the REDUCE trial. Curr Opin Urol 2005; 15: 29-32.

13 Andriole G, Bostwick D, Gomella L, Marberger M, Montorsi F, Pettaway $\mathrm{C}$ et al. Further analyses from the REDUCE Prostate Cancer Risk Reduction Trial. J Urol 2009; 181 (Supplement): 555.

14 Men Silent About Cancer-Until Women Step In. Men's Health Survey sponsored by Gilette. Press release, 16 June 2006 http://www.prostatecancerfoundation.org/site/c.itIWK2OSG/ b.1778841/k.6564/PR June 16 2006.htm

15 Patient survey shows most popular treatments, side effect prevalence and patient preferences for information. Annual Prostate Cancer/Men's Health Survey Released. 6 May 2006 http://www.fightprostatecancer.org/site/News2?page = NewsArticle\&id $=6929$

16 Fitzpatrick J, Corcoran N, Fitzpatrick JM. Prostate cancer: how aware is the public? Br J Urol 1998; 82: 43-48.

17 Schulman C, Kirby R, Fitzpatrick J. Awareness of prostate cancer among the general public: findings of an independent international survey. Eur Urol 2003; 44: 294-302.

18 Johns LE, Houlston RS. A systematic review and meta-analysis of familial prostate cancer risk. BJU Int 2003; 91: 789-794.

19 SEER database. (http://seer.cancer.gov/statfacts/html/prost.html)

20 Moulder JE, Foster KR, Erdreich LS, McNamee JP. Mobile phones, mobile phone base stations and cancer: a review. Int J Radiat Biol 2005; 81: 189-203.

21 Schröder FH, Hugosson J, Roobol MJ, Tammela TL, Ciatto S, Nelen $\mathrm{V}$ et al. Screening and prostate-cancer mortality in a randomized European study. N Engl J Med 2009; 360: 1320-1328. 


\section{Appendix A}

Survey questionnaire

Consumer survey questionnaire-final (11/10/07)

\section{ASK ALL}

Q1 Which one of the following is the most common form of cancer in men in this country?

ROTATE. ONLY ONE ANSWER POSSIBLE. Prompted

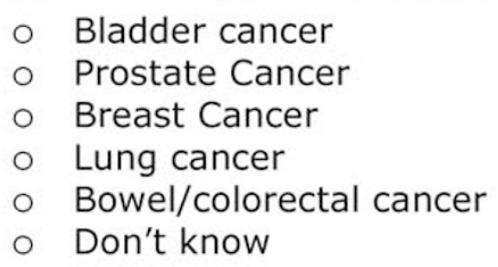

\section{WELL SAMPLE 2: ASK ALL WELL MALE/ WELL FEMALE}

Q2 As far as you know, does Prostate Cancer only affect $<<$ men $>>$, only affect $<<$ women $>>$ or both?

ROTATE $<<$ TEXT $>>$. ONLY ONE ANSWER POSSIBLE

Only Men

Only Women

Both

Don't know

\section{ASK ALL}

Q3 Which one of the following is more common in this country, << breast cancer in women $>>$ or $<<$ prostate cancer in men $>>$ ?

ROTATE $<<T E X T>>$. ONLY ONE ANSWER POSSIBLE

\section{ASK ALL}

$$
\begin{aligned}
& \text { Breast cancer in women } \\
& \circ \text { Prostate cancer in men } \\
& \circ \text { Equally common } \\
& \circ \text { Don't know }
\end{aligned}
$$

Q4 In your opinion, which of the following factors, if any, could mean that a person becomes more likely to develop Prostate Cancer? Please answer yes or no to each of the following.

ROTATE. MORE THAN ONE ANSWER POSSIBLE

Family history/genetics

Age

Smoking

Drinking

Number of sexual partners

Any other factors you can think of? If yes, please specify

\section{ASK ALL}

Q5 And in your opinion, which of the following, if any, can men do to reduce their likelihood of developing Prostate Cancer? Please answer yes or no to each of the following.

ROTATE. MORE THAN ONE ANSWER POSSIBLE 
Exercise regularly

Eat plenty of fruit and veg

Have regular check-ups/screening

Not carrying a mobile phone in their pockets

Can you think of anything else? If yes, please specify

\section{ASK ALL}

IF PCa MALE (Sample 1 ): $\quad$ Q6 Before you were diagnosed with prostate cancer, how much at risk did you believe you were of developing Prostate Cancer?

IF PCa FEMALE (Sample 1): Q6 Before your husband/partner was diagnosed with prostate cancer, how much at risk did your husband/partner believe he was of developing Prostate Cancer?

If "Well" Male (Sample 2): $\quad$ Q6 Generally speaking, how much at risk of prostate cancer do you believe you are compared to an average man of the same age?

If "Well" Female (Sample 2): Q6 Generally speaking, how much at risk of prostate cancer do you believe your husband/partner is compared to an average man of the same age?

ONLY ONE ANSWER POSSIBLE Prompted

Very high risk

Quite high risk

Average risk

Low risk

Very low risk

\section{ASK ALL}

Q7 And why do you think that?

PLEASE BE AS SPECIFIC AS POSSIBLE. ANYTHING ELSE?

\section{WELL SAMPLE 2 (ASK IF WELL MALE/WELL FEMALE)}

Q8 In your opinion, among those who have ever suffered from Prostate Cancer, what percentage of them actually die from the disease, as opposed to old age or another cause of death? PLEASE GIVE YOUR BEST ESTMATE

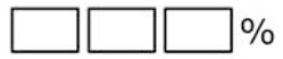

\section{ASK ALL}

Q9 Which, if any, of the following is used to screen for Prostate Cancer?

ROTATE, MORE THAN ONE ANSWER POSSIBLE. Prompted

Stool sample test

Sperm count

PSA test

CRP test

Something else/Other? If yes, please specify

Don't know 